\title{
Overall Review On Permeation Enhancers in Drug Delivery
}

\section{Systems}

Ganparaju Vaishnavi and Abbaraju Krishna Sailaja*

Department of Pharmaceutics Rbvrr Womens College of Pharmacy, Affiliated to Osmania University, Hyderabad.

*Corresponding author: Abbaraju Krishna Sailaja, Department of Pharmaceutics Rbvrr Womens College of Pharmacy, Affiliated to Osmania University, Hyderabad.

Received date: May 04, 2021; Accepted date: May 11, 2021; Published date: May 17, 2021

Citation: Ganparaju Vaishnavi and Abbaraju Krishna Sailaja, (2021) Overall Review On Permeation Enhancers in Drug Delivery Systems. J. Archives of Medical Case Reports and Case Study. 4(3); DOI:10.31579/2692-9392/051

Copyright: (c) 2021 Abbaraju Krishna Sailaja, This is an open access article distributed under the Creative Commons Attribution License, which permits unrestricted use, distribution, and reproduction in any medium, provided the original work is properly cited.

\section{Abstract}

$60 \%-70 \%$ Several drugs can be administered orally as liquids, capsules, tablets, or chewable tablets because the oral route is the most convenient, safest and less expensive. The important challenge in the oral drug delivery is the growth of novel approaches to approve absorption of poorly permeable drugs across the intestinal permeability. The Biopharmaceutics Classification System (BCS) categorized based on their solubility and permeability. The BCS Class III, Class IV with low permeability across the biological membranes with low bioavailability. While these drugs are pharmacologically effective, poor absorption due to low permeability becomes the rate-limiting step to improve oral bioavailability. Various approaches for improving the permeability include physical, chemical, colloidal carriers and other methods such as prodrugs, permeation enhancers, ion-pairing, nanoencapsulation and combination/hybridization of one or more traditional approaches to improve drug permeability for better absorption. Among many advantages over other routes of administration 3 crucial ones are avoiding metabolism in liver, minimal negative effects and increased bioavailability. This article discusses the commonly various strategiesand various approaches for improving the permeability of BCS Class III and Class IVdrugs to enhance bioavailability.

Keywords: various strategies for permeability enhancement techniques; various approaches to improve permeability

\section{PERMEABILITY ENHANCEMENT TECHNIQUES:}

Bile Salts:

Bile, which includes glycine and taurine conjugates of cholic acid and chenodeoxycholic acid emulsifies dietary fat, accelerates lipolysis and transport of lipid products through the unmoved water layer of the intestinal mucosa by micellar solubilisation. The diminishing order of hydrophilicity is as follows: taurine conjugates greater than glycine conjugates greater than free bile salts. Polarity increases depending on the number of hydroxyl groups. Bile salts are capable to bind with calcium, their binding properties decreases with increasing hydrophilicity. A transcellular absorption improving effect is suggested by the phospholipid disarranging of unconjugated and conjugated bile salts. Colonic tight junction structure appears to be persuaded by low bile salt concentrations (5mM and lower) in rabbits and rats. This paracellular absorption influencing effect is suggested to be intermediated by binding of calcium. However, the suggested co-carcinogenic \& co-mutagenic effects of secondary bile salts intimidate the development of bile salts containing pharmaceutical formulations.

\section{Nano emulsions:}

Nano emulsions are excellent vehicles for oral delivery of poorly permeable and/or highly lipophilic drugs as they can be manufactured from excipients that have solubilising or even permeation enhancing properties. Alike to conventional emulsions, they also improved gastrointestinal absorption and reduce inter $\&$ intraindividual unevenness for a variety of drugs. Moreover Additionally, due to their very large interfacial area, they display excellent drug release properties. The Pluronics ${ }^{\circledR}$, also specified as poloxamersare triblock copolymers of poly (oxy ethylene)-poly (oxy propylene)-poly (oxy ethylene) $[(\mathrm{EO}) \mathrm{x}(\mathrm{PO}) \mathrm{y}(\mathrm{EO}) \mathrm{x}]$. They are predominantly used as solubilisers, wetting agent for microemulsions and as micro container for drugs after micellization. It has been established that Pluronics ${ }^{\circledR}$ may influence the carrier-mediated transport of drugs depending on their structural composition. This effect might be beneficient for the treatment of drugresistant tumors as well as to improve the oral bioavailability of actives. Additionally, since the Pluronics ${ }^{\circledR}$ are known to obstruct p-gp-mediated drug efflux, they might also be used for formulation of actively effluxed drugs like many cytostatics $[1,2]$.

\section{Cyclodextrin inclusion complex:}

Cyclodextrins (CDs) are cyclic oligosaccharides consisting of $\mathrm{d}(+)$ glucopyranose units, attached by $[1,4]$ glucosidic bonds. They have an intrinsic capability of forming inclusion complexes with a variety of molecules owing to their special structures, with a hydrophilic outer surface and a hydrophobic cavity lined with protons. CDs have the ability 
to interact with solubility drugs and drug candidates resulting in an increase in the drug's apparent water solubility and dissolution rates. CD complexation improves oral bioavailability of poorly solubility drugs. The increase in solubility also affects dissolution rate and improves oral bioavailability. Eg. Exemestane is a neutral substance with steroidal structure categorize by high lipophilicity. It is freely soluble in $\mathrm{N}, \mathrm{N}$ dimethyl formamide, soluble in ethanol and insoluble in water $(80 \mu \mathrm{g} / \mathrm{ml})$. This is an encouraging approach to improve the poor bioavailability of EXE, which is commonly used for longer period oral administration in post-menopausal breast cancer chemotherapy.

\section{Spray freeze dying:}

Spray freeze drying is also another technique, which can be used for enhancement of permeability e.g. Spray freeze drying of oleonolic acid (OA) is a BCS class 4 compound polyvinyl pyrolidone-40 (PVP-40) acts as stabilizer and sodium caprate (CS) as wetting agent and penetration enhancer produces kinetically stable, amorphous solid dispersion system with invitro dissolution performance and better and more even absorption in comparison with commercial OA tablet. The SFD processed OA formulation and commercial OA tablet generally exhibit large interanimal variability in oral bioavailability, compatible with the absorption of BCS class IV compounds which are poorly permeable drugs. Inclusion of SC coupled with the substitute of OA with its sodium salt (OA-Na) in the formulation shows significant decrease in the observed absorption inconsistency $[3,4]$.

\section{Chitosan derivatives:}

Chitosan is a non-toxic, biocompatible polymer, which having several applications in drug delivery including that of absorption of hydrophilic macromolecular drugs. Chitosan, when protonated ( $\mathrm{pH}$ 6.5), is able to enhance the paracellular permeability of peptide drugs across mucosal epithelia. TMC (trimethyl chitosan chloride) enhance the permeation and $r$ absorption of neutral and cationic peptide across intestinal epithelia. The mechanism by, which TMC enhances intestinal permeability is same to that of protonated chitosan. It reversibly interacts with compounds of tight junctions, leading to paracellular routes.

\section{Saponins:}

Saponins, which are glycosides of vegetable source with surface tension reduces properties and haemolytic action. They are capable of precipitating sterols and intestinal and transdermal absorption. It is imaginable that the absorption supporting properties of saponins are mediated by their surfactants properties. On the other hand, a transcellular promoting effect may caused by interaction with the membrane stabilizer cholesterol. This shows that saponins exhibit absorption promoting activity at low concentrations. However; also for these compounds the issue of safety against efficacy requires further examine.

\section{Straight chain fatty acids:}

The medium chain fatty acids involve capric acid (C10), lauric acid (C12) and long chain fatty acids, such as oleic acid (C18) acts as permeation enhancer to increase the permeability of a group of hydrophilic drugs by dilating the tight junction and/or changing the cytoskeleton of the intestinal epithelial cells without prominent cytotoxicity. One of the benifit of these excipients is incorporating into the conventional oral dosage forms without expensive formulation technique.

\section{Self-micro-emulsifying drug delivery systems (SMEDDS):}

The GI absorption of poorly permeable drugs i.e. BCS class 4 drugs can be improved by using self-microemulsifying drug delivery systems. Generally, these systems are isotropic mixtures of oils, surfactants and cosolvents /co-surfactants. As administered into the GI system, they are diluted with gastrointestinal fluid and the gastric motility provides the agitation for the formation of a fine oil-in-water $(\mathrm{o} / \mathrm{w})$ micro emulsion (SMEDDS).

\section{Self-double emulsifying drug delivery system (SDEDDS):}

The self-double emulsifying drug delivery systems (SDEDDS) can be used for improving oral bioavailability of drugs with high solubility and low permeability BCS Class III, but their industrial application is sufficient because of low stability. SDEDDS emulsify to water-in-oil-inwater $(w / o / w)$ double emulsion in the diversified aqueous gastrointestinal nature, with drugs encapsulated in the internal water phase of the double emulsions. The internal aqueous droplets encapsulated by the oil membrane for hydrophilic drugs. This structure can safeguard the drug dissolved in the internal aqueous phase and promising for improving oral bioavailability of compounds $[5,6]$.

\section{PERMEATION ENHANCERS}

Permeation enhancers used to enhance membrane transport of proteins, peptides and other physical, chemical, colloidal compounds across biological membranes.

\section{VARIOUS APPROACHES}

\section{Physical approach \\ 2. Chemical approach \\ 3. Colloidal carriers}

Physical approach: Physical enhancers interact physically with the skin in various ways and damage the skin integrity to open the skin pores. The combination of iontophoresis with permeation enhancers was found to be promising in drug delivering.

$>$ Iontophoresis: In Iontophoresis the drug is located on the skin with an electrode having the same charge as that of the drug molecules and a current of few milliampere $(<0.5 \mathrm{~mA})$ is applied, the electrode decline the drug molecules of the same charge through the skin to the electrode of opposite charge and drug delivers at a rapid rate. Iontophoresis can be used in combination with chemicals, electrophoresis, or ultrasound for better results. 
Eg: In this study, they explored intestinal iontophoresis mucoadhesive patches as a novel method to facilitate permeation of insulin across both invitro and in vivo. It was conducted by using insulin as a model drug and was found to remarkably improve insulin transport across intestine both in vitro and its pharmacodynamics in vivo. A 35\% drop in Trans Epithelial Electrical Resistance (TEER) was observed in Caco2 monolayers culture in trans wells subjected to iontophoresis that consequently led to almost three-fold enhancement in insulin transport across the cell. In rats, insulin loaded mucoadhesive patches $50 \mathrm{U} / \mathrm{Kg}$ integrated with iontophoresis electrical circuit and surgically placed in the intestine, led to profound Hypoglycaemic that depends on insulin dose and current density. In addition, iontophoresis did not cause any structural damage to intestinal tissues, indicating that the technology is safe to use.

$>$ Sonophoresis: In Sonophoresis, the sound waves of frequency $20 \mathrm{KHz}-10 \mathrm{MHz}$ are applied for 15 seconds to the skin with the drug which delivers the drug through the skin by fracturing the lipid bilayer. The drug is mixed with a coupling agent transmits the sound waves through the skin along with the drug.this approach results in direct entry of bioactive molecules into the systemic circulation, avoiding first-pass metabolism, efflux transporters, digestive enzymes and unfavorableconditions associated with other oral administration .

eg: Tizanidine is a suitable model drug for transdermal drug delivery. The best permeation enhancement was obtained with a combination of citral and ethanol, which also enabled adequate and controlled delivery of TIZ through the skin for $24 \mathrm{hr}$. The permeation of TIZ over $30 \mathrm{~min}$ duration was increased by applying ultrasound in connection with the combination of citral and ethanol. The amount of permeated drug was again significantly $(\mathrm{p}<0.05)$ higher than observed for passive diffusion of TIZ formulated in 50\% ethanol containing $5 \% \mathrm{v} / \mathrm{v}$ citral $(0.087 \pm 0.014$ at $15 \mathrm{mins}$ and $0.112 \pm 0.040 \mathrm{mg}$ at $30 \mathrm{~min}$, respectively).

\section{Chemical approaches [7]:}

Antihypertensive agent through the skin by chemical permeation enhancers and iontophoresis for improving permeation. The combination of iontophoresis with permeation enhancers was found to be promising in delivering atenolol across the skin, and the highest permeation was obtained when oleic acid acts as chemical enhancer was combined with iontophoresis. The in vitro flux value obtained (when oleic acid combined with iontophoresis) was $0.71 \mathrm{mg} / \mathrm{cm} 2 / \mathrm{h}$, which fact that a therapeutically effective concentration can be obtained with $1.2 \mathrm{~cm} 2$ of skin area.

\section{Multiple emulsion or double emulsion:}

Multiple emulsion systems are novel developments and promising oral administration. Multiple emulsion termed as emulsions of emulsions, in which globules of the dispersed phase encapsulates smaller droplets, consists of liquid miscible with and in some cases identical with continuous phase. This is made possible by double emulsification, hence called as double emulsion. It has both hydrophilic and lipophilic emulsifiers are used for the formation of multiple emulsions.

Double emulsions are helpful to maintain drug concentrations with therapeutic range in lymph vessels. The transport of drugs to the mesenteric lymph nodes rather than into the portal system directly is due to encapsulation of the drug by the oil phase, which provides more lipophilic nature therefore, are absorbed through lymphatic vessels. In addition, w/o/w emulsions might act as carriers for the delivery of polypeptide/protein drugs, which require both protection from the gastric fluids and delivery via lymph nodes

Eg:The study was to develop water-in-oil-in-water (w/o/w) type Multiple Emulsion of Lisinopril dihydrate (BCS Class -III Drug) for improving its oral bioavailability via improving permeation .In vitro drug diffusion study was done through dialysis membrane showed rapid release in beginning and then slower release of drug in 8 hours. Permeability study showed higher permeation rate from Multiple emulsion than plain drug solution and ex vivo permeability studies were performed in Franz diffusion cell using rat intestine.

\section{Solid lipid nanoparticles (SLN):}

Solid lipid nanoparticles (SLN) are aqueous colloidal dispersions, the matrix of which comprises of solid biodegradable lipids. Colloidal carriers, such as emulsions, liposomes and polymeric micro- and nanoparticles.Solid lipid nanoparticles (SLNs) are appealing extensive notice because of showing increased solubility and improved oral bioavailability via different mechanisms [8].

Eg:- A study was conducted to compare and peruse the in-situ permeation of nebivolol (NBV) loaded SLN and its commercial tablet formulation used for the treatment of hypertension. For this aim Single-Pass Intestinal Perfusion (SPIP) method was used for in-situ permeation studies. PEG modified SLN can be used to enhance oral absorption of NBV, and SLNs alone can be used as permeation enhancer in oral drug delivery.In perfusion studies, metoprolol tartrate was used as a high permeability reference compound in order to compare the permeability coefficient of pure NBV and NBV in tablet.

\section{Prodrugs:}

Prodrugs are utilized to improve permeability of drug by improving its lipophilicity.Prodrugsacts as a false carrier after entering into biological membrane it gets to its Original form and release the drug. In order to improve lipophilicity, and thus passive transport through biological membranes, compounds containing polar or ionizable groups can be converted into ester prodrugs.

Novel lipophilic ester prodrug approach, combined with oral lipid-based formulation, to efficiently deliver lopinavir (LPV) to the mesenteric lymph and MLNs. A number of prodrugs was designed using an in-silico model for prediction of affinity to chylomicrons (CMs), and then synthesized. Subsequently, LPV and selected prodrug candidates were evaluated for their in vivo pharmacokinetics and biodistribution in rats. A combination of the lipophilic prodrug approach with lipid-based formulation resulted in efficient targeting of LPV to HIV reservoirs in mesenteric lymph and MLNs. The maximum levels of LPV in mesenteric lymph were 1.6- and 16.9-fold higher than protein binding-adjusted $\mathrm{IC}_{90}$ (PA-IC90) of LPV for HIV-1 (140 ng/mL) following oral administration of simple alkyl ester prodrug and activated ester prodrug, 
respectively. Moreover, the concentrations of LPV in MLNs were 1.1and 7.2-fold higher than PA- $\mathrm{IC}_{90}$ following administration of simple alkyl ester prodrug and activated ester prodrug, respectively. Furthermore, the bioavailability of LPV was also increased ester prodrug compared to unmodified LPV. This approach, especially if can be translated to other antiretroviral drugs, has potential for reducing the size of HIV reservoirs within the mesenteric lymphatic system.

\section{CONCLUSION:}

Permeability issues as well as drug resistance which can be overcome with novel approaches such as nanoparticles, microemulsions, lipoproteins which led to increase permeability membrane. Some advantageous techniques like bile salts, surfactants, chelating agents, cyclodextrin, nanoemulsion, fatty acids, SMEDDS, SDEDDS are promising techniques to enhance permeability to enhance oral bioavailability. Also some approaches like physical, chemical permeation enhancers used to enhance permeability of drug when combined with iontophoresis for better absorption. This review focused on various permeability techniques to improve permeability of drug and various approaches to enhance permeability of drug to enhance bioavailability. The Biopharmaceutics Classification System (BCS) categorized based on their solubility and permeability. The BCS Class III, Class IV with low permeability across the biological membranes with low bioavailability.

\section{REFERENCES:}

1. Shaikh MS I, Nikita D. Derle and Rajendra Bhamber,et al. Permeability Enhancement Techniques for Poorly Permeable Drugs: A Review, 2012, journal of pharmaceutical science,
2. Dinesh M Damecha, Vivek B,Amit A, et al. Physical Approaches to Penetration Enhancement, 2010, international journal of health research,

3. Vikas Jhawat, et al. Current drug therapy, Recent Advances in Permeation Enhancement Techniques for Transdermal Drug Delivery Systems: A Review,

4. Amrita Banerjee, Renwei Chen, Shamsul Arafin, Samir Mitragotri, et al. Intestinal iontophoresis from mucoadhesive patches: a strategy for oral delivery, 2019,

5. Srinivas Mutalik, Harendra S. Parekh, Nigel M. Davies \&Udupa Nayanabhirama, et al. A combined approach of chemical enhancers and sonophoresis for the transdermal delivery of tizanidine hydrochloride, 2008,

6. Sawant kk, et al. Development and Optimization of $\mathrm{w} / \mathrm{o} / \mathrm{w}$ Multiple Emulsion of Lisinopril Dihydrate Using Plackett Burman and Box-Behnken Designs, journal of Nanomedicine and Nanotechnology,2017,

7. Evren Homan GÖKÇE, Mustafa Sinan KAYNAK, Aysu YURDASIPER , Neslihan ÜSTÜNDAĞ-OKUR , Selma ŞAHIN , Comparison of intestinal permeability of nebivolol hydrochloride loaded solid lipid nanoparticles with commercial nebivolol tablet, Marmara pharmaceutical Journal, 2018

8. Chaolong Qin, Yenju Chu, Wanshan Feng, et al. Targeted delivery of lopinavir to HIV reservoirs in the mesenteric lymphatic system by lipophilic ester prodrug approach, 2020, National library of medicine
This work is licensed under Creative Commons Attribution 4.0 License

To Submit Your Article Click Here: Submit Article
Ready to submit your research? Choose Auctores and benefit from:

* fast, convenient online submission

* rigorous peer review by experienced research in your field

* rapid publication on acceptance

* authors retain copyrights

* unique DOI for all articles

* immediate, unrestricted online access

At Auctores, research is always in progress.

Learn more www.auctoresonline.org/journals/archives-of-medical-casereports-and-case-study 